\title{
Ubber das asymptotische Verhalten der Lösungen einer linearen Differentialgleichung mit Nachwirkung
}

\author{
K. Morgentihat
}

In der Aṛbeit werden für die Differentialgleichung

$$
x^{\prime}(t)=-\int_{z=0}^{\infty} x(t-\tau) d r(t, \tau)
$$

notwendige Bedingungen und hinreichende Bedingungen für die Existenz final positiver Lösungen angegeben, die bekannte Bedingungen verallgemeinern. Ihre final positiven Lösungen werden mit Hilfe von Lösungen der entsprechènden Differentialungleichungen abgeschätzt. Abschließend wird ein Entwicklungssatz bewiesen. Die Resultate beruhen hauptsächlich auf der Betrachtung der Lösungen der Differentialungleichungen und der Anwendung von Vergleichssätzen für diese Lösungen.

В работе для дифференциального уравнеиия

$$
x^{\prime}(t)=-\int_{t=0}^{\infty} x(t-\tau) d r(t, \tau)
$$

даются необходимые условия и достаточные условия для существования финально положительных решений, которые обобщают иввестные условия. Его финально положительные решения оцениваются с помощью решений соответствующих діифференциальных неравенств..В заключение доказывается теорема разложения. Результаты основаны главинім образом на рассмотрении ренений дифференциальных неравенств и на применении теорем сравнсния для әтих решений.

In the paper for the differential equation

$$
x^{\prime}(t)=-\int_{\tau=0}^{\infty} x(t-\tau) d r(t, \tau)
$$

necessary conditions and sufficient conditions for the existence of eventually positive solutions are given which generalize known conditions. Their eventually positive solutions are estimated by means of solutions of the related differential inequalities. Finally an expansion theorem is proved. The results are mainly based on the consideration of the solutions of the differential inequalities and the application of comparison thérems for these solutions.

\section{Einleitung}

Wir betrachten die Differentialgleichung

$$
x^{\prime}(t)=-\int_{t=0}^{\infty} x(t-\tau) d r(t, \tau) \quad:(-\infty<A \leqq t)
$$

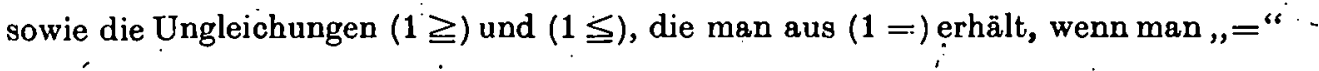




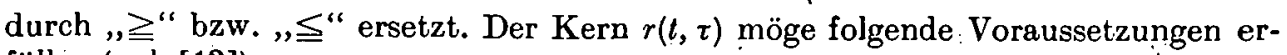
füllen (vgl. [13]):

1. $r(t, \tau)$ ist definiert für $A \leqq t, 0 \leqq \tau$.

2. Es gilt $r(t, 0)=0(A \leqq t)$, und $r(t, \tau)$ ist bei jedem $t \in[A, \infty)$ monoton wachsend in $\tau$.

3. Es existiert eine áuf $[A, \infty)$ stetige Funktion $\sigma(t) \geqq 0$, so daß gilt:

3.1. $r(t, \tau)=r(t, \sigma(t))(A \leqq t ; \sigma(t) \leqq \tau)$,

3.2. $r(t, \sigma(t))$ ist auf $[A, \infty)$ stetig, $\min \left(\sigma(t), \sigma\left(t^{\prime}\right)\right)$

3.3. $\lim _{\substack{t^{\prime} \rightarrow t^{\prime} \\ A \leqq t^{\prime}}} \int_{r=0}\left|r(t, \tau)-r\left(t^{\prime}, \tau\right)^{\prime}\right| d \tau=0(A \leqq t)$.

Unter den genannten Voraussetzungen úntersuchen wir das asymptotische Verhalten der Lösungen von $(1=)$. Diese Gleichung ist intensiv untersucht worden. Wir verweisen dazu insbesondere auf MYŠKrs [13] und Kozakiewicz [1-8]. In der vorlicgenden Arbeit knüpfen wir an die Arbeiten [7, 8] des letzteren an, deren Ergebnisse wir wesentlich benutzen. Unsere Resultate beruhen hauptsächlich auf der Betrachtung der Lösungen der Differentialungleichungen ( $1 \leqq)$ und ( $1 \geqq$ ) und der Anwendung 'von Vergleichśätzen für diese Lösungen.

Zunächst beschäftigen wir uns mit der Frage, wann $(1 .=)$ nichtoszillierende Lösungen besitzt bzw. wann alle Lösungen von $(1=)$ oszillieren. Es gibt, eine Vielzahl von Arbeiten, in denen diese Frage für Spezialfälle von $(1=)$ untersucht wurde. Wir verweisen zum Beispiel auf Iadaas; Lakshmikantham und Papadaris [9], Ladas [10], Ladde [11], Tramov [14] sowie auf den Utbersichtsartikel ŠEvelo und Varech [15]. Der allgemeine Fall wurde in MYškis [13] behandelt, wo auch die Vermutung ausgesprochen wurde, daß sich die dort angegebenen Kriterien verbessern lassen. Wir geben hier nun notwendige Kriterien und hinreichende Kriterien für die Existenz final positiver Lösungen von $(1=)$ an, die die in [ $13:$ Kap. VI] angegebenen Kriterien verallgemeinern. Die hinreichenden Bedingungen erhalten wir alle durch Anwendung unseres Satzes $3: .(1=)$ besitzt genau dann eine positive Lösung, wenn $(1 \leqq)$ eine positive Lösung besitzt.

Im zweiten Teil der Arbeit schätzen wir die final positiven Lösungen von $(1=)$ mit Hilfe bestimmter Lösungen der Ungleichungen $(1 \leqq)$ und $(1 \geqq)$ ab. Dabei gelingt es auch, die Existenz der entsprechenden Grenzwerte nachzuweisen. Die angewandten Hilfssätze (Lenıma 4-6) sind von selbständigem Interesse und auch in anderen $\mathrm{Zu}$ sammenhängen nützlich. Bei Verwendung anderer Lösungen von $(1 \leqq)$ und $(1 \geqq)$ als - Vergleichsfunktionen gestatten sie es, Analoga zu unseren Resultaten zu erhalten.

Die erwähnten Lemmata erlauben es im letzten Abschnitt zu zeigen, da $B 0<n(\infty)$, $N(\infty)<\infty$ hinreichend ist für die Gültigkeit des Entwicklungssatzes (vgl. dazu $[3,13])$.

$\therefore$ Viele Resultate der vorliegenden Arbeit lassen sich auf die Gleichung

$$
x^{\prime}(t)=\alpha(t) x(t)-\int_{i=0}^{\infty} x(t-\tau) d r(t, \tau) \quad(-\infty<A \leqq t)
$$

übertragen, wobei $\alpha(t)$ eine stetige Funktion beliebigen Vorzeichens ist. Wir kommen darauf in einer späteren Arbeit zurück. Bei der Untersuchung anderer FunktionalDifferentialgleichungen ist unsere Methode ebenfalls anwendbar. 


\section{Vergleichssätze}

Wegen der Bedingung 3.1 reduziert sich das Integral in $(1=)$ auf ein endliches Integral mit der óberen Grenze $\sigma(t)$, das also nur aus formalen Gründen als uneigentliches

- Jntegral geschrieben wurde. Genauer, sei bei $A \leqq t$

$$
\Delta(t)=\inf \{\tau: 0 \leqq \tau, r(t, \tau)=r(t, \sigma(t))\} .
$$

Dann gilt $0 \leqq \Delta(t) \leqq \sigma(t), r(t, \tau)=r(t, \sigma(t))(\Delta(t)<\tau)$ und

$$
\int_{\tau=0}^{\infty} x(t-\tau) d r(t, \tau)=\int_{\tau=0}^{\Delta(t)} x(t-\tau) d r(t, \tau)+x(t-\Delta(t))[r(t, \sigma(t))-r(t, \Delta(t))] .
$$

Der Wert des Integrals $\int_{i=0}^{\infty} x(t-\tau) d r(t, \tau)$ hängt also nur von den Werten von $x$ in [ $t-\Delta(t), t]$ ab. Betrachten.wir alle $t \geqq T$, so hängen die Werte des Integräs für diese $t$ also nur von den Werten von $x$ in Punkten $s \geqq \inf \{t-\Delta(t): t \geqq T\}$ ab.

Wegen des eben Gesagten definieren wir für $\bar{T} \geqq \boldsymbol{A}$

$$
-E(T)=\inf \{t-\Delta(t): t \geqq T\} .
$$

Offenbar gilt entweder $E(T) \equiv-\infty$ ' $(A \leqq T)$ oder $E(T)>-\infty(A \leqq T)$.'Wegen $\Delta(t) \geqq 0$ ist $E(T) \leqq T(A \leqq T)$ und bei $A \leqq T_{1} \leqq T_{2}$ gilt $E\left(T_{1}\right) \leqq E\left(T_{2}\right)$. $\mathscr{E}\left(T^{\prime}\right)$ $(A \leqq T)$ sei im Fall $E(T)>-\infty$ das Intervall $\left[E\left(T^{\prime}\right), T^{\prime}\right]$ und im Fall $E(T)=-\infty$ das Intervall $(-\infty, T]$.

Unter einer Lösung von $(1 \leqq)$ verstehen wir eine auf $\mathscr{E}(A) \cup[A, \infty)$ definierte stetige reellwertige Funktion, die auf $[A, \infty)$ differenzierbar ist und der Ungleichung $(1 \leqq)$ geniigt. Analog verwenden wir den Lösungsbegriff bezüglich $(1 \geqq)$ und $(1=)$. Unter der Ableitung an der Stelle $A$ verstehen wir immer die rechtsseitige Ableitung. Bei den angegebenen Voraussetzungen existiert zu jeder auf $\mathscr{E}(A)$ stetigen Funktion $\varphi$ genau eine Lösung $x$ vòn $(1=)$, für die $x(t)=\varphi(t)(t \in \mathscr{E}(A))$ gilt (s. [13: Th. 1]).

Wir benötigen im weiteren die folgenden zwei Vergleichssätze.

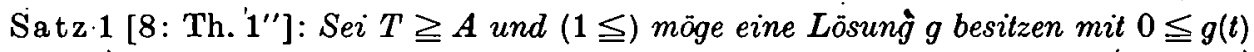
$(t \in \mathscr{E}(T))$ und $0<g(t)(T \leqq t)$. Ist dann f eine Lösung von $(1 \geqq)$ mit $f(t) \leqq 0(t \in \mathscr{E}(T))$ und $f(T)=0$, so gill $0 \leqq f(t)(T \leqq t)$.

Zusatz: Ist $0<f^{\prime}\left(I^{\prime}\right)$, so gilt $0<f(t)(\dot{T}<t)$.

Bewe is:- Wegen $f(T)=0$ und $0<f^{\prime}(T)$ können wir ein $T_{1}>T$ wählen, so daß

$$
0<f(t) \quad\left(T<t \leqq T_{1}\right)
$$

gilt. Weiter können wir ein $\gamma>0$ wählen, so daß $0<\gamma g\left(T_{1}\right)<f\left(T_{1}\right)$ ist. Sei nun $T_{2}=\inf \{t: T<t$ und $\gamma g(t)<f(t)\}$. Dann ist $T<T_{2}<T_{1}$ und es gilt

$$
f(t) \leqq \gamma g(t) \quad\left(t \in \mathscr{E}\left(T_{2}\right)\right), \quad f\left(T_{2}\right)=\gamma g\left(\dot{T_{2}}\right) .
$$

Also erhalten wir, Satz 1 auf die Lösung $\tilde{f}=f-\gamma g$ von $(1 \geqq)$ anwendend,

$$
f(t) \geqq \gamma g(t)>0 \quad\left(T_{2} \leqq t\right) .
$$

Aus (2) und (4) folgt die Behauptung

Aus Satz 1 ergibt sich der folgende Satz 2 (s. [8: Th. 2]). 
Satz 2: Seif eine Lösung von $(1 \geqq)$ und g eine Lösung von $(1 \leqq)$. Weiter sei $A \leqq T$, $0 \leqq g(t)(t \in \mathscr{E}(T)), 0<g(t)(T \leqq t), f(t) \leqq g(t)(t \in \mathscr{E}(T))$ und $f(T)=g\left(T^{\prime}\right)$. Dann ist $q(t)=\frac{f(t)}{g(t)}$ auf $[T, \infty)$ monoton wachsend.

\section{Existenz.final positiver Lösungen von $(1=)$}

Wir beschäftigen uns mit der Frage nach der Existenz nichtoszillierender Lösungen von $(1=)$. Da mit $f$ auch $-f$ eine Lösung von $(1=)$ ist, können wir o. B. d. A. nach der Existenz final positiver Lösungen von $(1=)$-fragen.

Eine auf $\mathscr{E}(A) \cup[A, \infty)$ definierte Funktion $f$ nennen wir final positiv, wenn ein $P$ existiert mit $0<f(t)(P \leqq t)$. Wir nennen $f$ positiv, wenn für alle $t \in \mathscr{E}(A) \cup[A, \infty)$ gilt $0<f(t)$. Analog verwenden wir die Bezeichnungen final negativ und negativ. $f$ heißt final monoton fallend, wenn ein $P$ existiert, so daß $f$ auf $[P, \infty)$ monoton fällt. $f$ heißt oszillierend, wenn $f$ in jedem Intervall $[P, \infty)$ Nullstellen besitzt, andernfalls heißt $f$ nichtoszillierend.

Zunächst formulieren wir den folgenden Satz 3, der unmittelbar aus dem Vergleichssatz 2 folgt.

Satz 3: $\left(1{ }^{\prime}=\right)$ ' besitzt genau dann eine positive Lösung, wenn.$(1 \leqq)$ eine positive Lösung besitzt.

Beweis: Da jede Lösung von $(1=)$ auch eine Lösung von $(1 \leqq)$ ist, genügt es zu zeigen, da $B$ die Existenz einer positiven Lösung $g$ von $(1 \leqq)$ die Existenz einer positiven Lösung $f$ von $(1=)$ nach sich zieht. Dazu betrachten wir diejenige Lösung $f$ von $(1=)$, für die gilt $f(t)=g(t)(t \in \mathscr{E}(A))$. Nach Satz 2 ist $f(t) \geqq g(t)>0 .(A \leqq t)$. Also ist $f$ eine positive Lösung von $(1=)$

Wenn in konkreten Fällen die Existenz positiver Lösungen von $(1=)$ nachgewiesen werden soll, so genügt es also, die Existenz einer positiven Lösung von $(1 \leqq)$ zu zeigen: Da die Lösungsmenge von $(1 \leqq)$ größer ist als diejenige von $(1=)$, ist dies häufig einfacher als der direkte Nachweis der Existenz positiver Lösungen von $(1=)$. Bevor wir Satz 3 anwenden, führen wir einige Bezeichnungen ein (vgl. [13]). Es sei

$$
\begin{aligned}
& \delta(t)=\sup \{\tau: 0 \leqq \tau \leqq \sigma(t), r(t, \tau) \leqq 0\}, t \in[A, \infty) \\
& \delta_{0}=\inf \{\delta(t): A \leqq t\} ; \Delta_{0}=\sup \{\Delta(t): A \leqq t\} \\
& M(t)=\left\{\begin{array}{l}
0 \text { bei } t<A \\
r(t, \sigma(t)) \text { bei } A \leqq t ;
\end{array}\right. \\
& m_{0}=\inf \{M(t): A \leqq t\} ; M_{0}=\sup \{M(t): A \leqq t\}
\end{aligned}
$$

Nach Voraussetzung 3.2 ist die Funktion $M$ in allen Punkten $t \neq A$ stetig und in $t=A$ rechtsseitig stetig. Die Funktionen $\delta$ und $\Delta$ können selbst bei ,gutartigen“" Kernen unstetig sein (s. [13]). Weiter sei bei $A \leqq T$

$$
\begin{aligned}
& n^{\prime}(T)=\inf \left\{\int_{t-\delta(t)}^{t} M(s) d s: T \leqq t\right\} \\
& N(T)^{\circ}=\sup \left\{\int_{t}^{t} M(s) d s: T \leqq t\right\}
\end{aligned}
$$


sowie

$$
\begin{aligned}
& n(\infty)=\lim _{T \rightarrow \infty} n(T)=\underline{\lim _{t \rightarrow \infty}} \cdot \int_{t-\delta(t)}^{t} M(s) d s ; \\
& N(\infty)=\lim _{T \rightarrow \infty} N(T)=\underset{\lim _{t \rightarrow \infty}}{\int} \int_{t-\Delta(t)}^{t} M(s) d s .
\end{aligned}
$$

Wir beträchten im folgenden oft die Funktion $V(\lambda)=\frac{\ln \lambda}{\lambda}$ für $0<\lambda \leqq \infty$. Dabei vereinbaren wir, daß $V(\infty)=0$ sein soll.

Sei nun $\psi$ eine auf $\mathscr{E}(A) \cup[A, \infty)$ definierte integrierbare und auf $[A, \infty)$ stetige Funktion. Weiter sei $g(t)=\exp \left[-\int_{A}^{t} \psi(s) d s\right], t \in \mathscr{E}(A) \cup[A, \infty)$. Dann ist $g$ auf $\mathscr{E}(A) \cup[A, \infty)$ positiv und stetig, auf $[A, \infty)$.differenzierbar und erfüllt offenbar genau dann $(1 \leqq)$, wenn

$$
\psi(t) \geqq \int_{\tau=0}^{\infty} \exp \left\{\int_{t=\tau}^{t} \psi(s) d s\right\} d r(t, \tau) \quad(A \leqq t)
$$

gilt. Wenn eine Funktion $\psi$ existiert, die (5) erfüllt, so besitżt $(1=)$ also eine positive Lösung. Hinreichend für (5) ist:

$$
-\psi(t) \geqq 0(t \in \mathscr{E}(A)), \psi(t) \geqq M(t) \cdot \exp \left\{\int_{t-\Delta(t)}^{t} \psi(s) d s\right\} \quad(A \leqq t) .
$$

Die folgenden Sätze erhält man bei spezieller Wahl von $\psi$.

Satz 4: Wenn eine Zahl $\hat{\imath} \geqq 0$ existiert, so da $\beta$

$$
\lambda \geqq \int_{\tau=0}^{\infty} \mathrm{e}^{\lambda \tau} d r(t, \tau) \quad(A \leqq \hat{t})
$$

gilt, so besitzt $(1=)$ eine positive Lösung.

Beweis: Wir setzen in (5) $\psi(t) \equiv \lambda$, das heißt, wir setzen $g(t)=\mathrm{e}^{-\lambda(t-A)}$

Hinreichend für $(6)$ ist $\lambda \geqq M(t) \mathrm{e}^{\lambda \triangleleft(t)}(A \leqq t)$.

Anmerkung: Ist die Differentialgleichung autonom, d. h., ist der Kern unabhängig von $t, r(t, \tau) \equiv r(\tau)$, so wird $(1=) \mathrm{zu}$

$$
x^{\prime}(t)=-\int_{\tau=0}^{0} x(t-\tau) d r(\tau), \quad \sigma=\text { konst, }
$$

und die Ungleichung (6) zu

$$
\lambda \geqq \int_{\tau=0}^{\sigma} \mathrm{e}^{\lambda_{\tau}} d r(\tau)
$$

(6a) hat genau dann, eine Lösung $\lambda \geqq 0$, wenn die Gleichung

$$
\lambda=\int_{r=0}^{\sigma} \mathrm{e}^{\lambda \tau} \dot{d r}(\tau)
$$

ène Lösung $\lambda \geqq 0$ besitzt. Satz 4 reduziert sich in diesem Fall also auf die einfache Aussage, daß $(1 \mathrm{a}=)$ eine positive Lösung besitzt, wenn (7) eine Lösung ' $\lambda \geqq 0$ hat. 
Dies ist natürlich richtig, denn (7) ist die charakteristische Gleichung zu $(1 \mathrm{a}=)$, die sich beim Ansatz $x(t)=\mathrm{e}^{-\lambda t}$ ergibt. Das folgende Kriterium wurde mir von E. Kozakiewicz mündlich mitgeteilt.

Satz 5: Ist $\int_{A}^{1} M(s) d s<1(A \leqq t)$, so hat $(1=)$ eine positive Lösung.

Beweis: Wir setzen $g(t)=1-\int_{A} M(s)$ as. Wegen der Voraussetzung ist $g(t)$ ständig positiv und man kann leicht nachprüfen, daß $g(1 \leqq)$ erfüllt. Im vorliegenden Fall ist $\psi(t)=\dot{M}(t) /\left(1-\int_{A}^{t} \dot{M}(s) d s\right)$

Satz 6: Wenn eine Zahl $c>0$ existiert, so daß

$$
\int_{\tau=0}^{\infty}\left(\exp \left[c \cdot \int_{t-1}^{i} M(s) d s\right]-c\right) d r(t, \tau) \leqq 0(A \leqq t)
$$

ist, so besitzt $(1=)$ eine positive Lösung.

Beweis: Wir setzen in (5) $\psi(t)=c \cdot M(t)$, d. h., wirsetzen $g(t)=\exp \left[-c \cdot \int_{A} M(s) d s\right]$. Wegen $M(t)=\int_{r=0}^{\infty} d r(t, \tau)(A \leqq t)$ kann man dann $(5)$ in der Form (8) schreiben

Hinreichend für $(8)$ ist $\exp \left[c \cdot \int_{t-\Delta(t)}^{t} M(s) d s\right]-c \leqq 0 \quad(A \leqq t), \mathrm{d} . \mathrm{H}_{\mathrm{i}}$,

$$
\int_{t-\Delta(t)}^{t} M(s) d s \leqq \frac{\ln c}{c}(A \leqq t)
$$

Ist $N(A) \leqq \frac{1}{\Theta}$, so existieren wegen $\max _{0<c<\infty} \frac{\ln c}{c}=\frac{1}{\mathrm{e}}$ Werte $c>0$, so daß

$$
\frac{\ln c}{c} \geqq N(A)=\sup \left\{\int_{t-\Delta(t)}^{-t} M(s) d s: A \leqq t\right\}
$$

gilt. Für diese $c$ ist also (9) erfüllt. Damit ist der folgende Satz 7 bewiesen.

Satz $7:$ Ist $N(A) \leqq \frac{1}{\mathrm{e}}$, so hat $(1=)$ eine positive Lösung.

Anmerkungen: 1. Die eben bezüglich des Punktes $A$ angewandte Schlußweise liefert allgemeiner, daß bei $\frac{\ln c}{c} \geqq N(T), T \geqq A$, die Funktion

$$
\varphi_{c}(t)=\exp \left[-c \int_{i}^{t} M(s) d s\right] .
$$

für $t \geqq T$ die Ungleichung $(1 \leqq)$ erfüllt. Analog kann man leicht nachprüfen, daß $\varphi_{c}$ bei $\frac{\ln c}{c} \leqq n(T), A \leqq T$, die Ungleichung $(1 \geqq)$ für $t \geqq T$ èfüllt. 
2. Wissen wir nur, daß für ein $T>A$ gilt $N(T) \leqq \frac{1}{\mathrm{e}}$, so erhalten wir nach Satz 7 nur, daß eine auf $\mathscr{E}(T) \cup[T, \infty)$ positive stetige Funktion existiert, die $(1=)$ für $t \geqq T$ erfüllt. Es bleibt dabei offen, ob auch eine positive Lösung 'existiert, die $(1=)$ für $t \geqq A$ erfüllt.

3. Nach [13: Th. 39] besitzt $(1=)$ bei $M_{0}<\infty, \Delta_{0}{ }^{-}<\infty, M_{0} \Delta_{0} \leqq \frac{1}{\mathrm{e}}$ eine positive Lösung. Dies ist in Satz 7 als Spezialfall enthalten. Da nämlich für alle $t \geqq A$ gilt $\int_{t=\Delta(t)}^{t} M(s) d s \leqq M_{0} \Delta_{0}$, folgt aus $M_{0} \Delta_{0} \leqq \frac{1}{\mathrm{e}}$ sofort $N(A) \leqq \frac{1}{\mathrm{e}}$. Also liefert Satz $7 \mathrm{die}$ Existenz einer positiven Lösung von $(1=)$.

Im folgenden setzen wir stets voraus

$$
\lim _{t \rightarrow \infty}(t-\Delta(t))=\infty
$$

Dann gilt $-\infty<E(t)(A \leqq t)$ und

$$
\lim _{t \rightarrow \infty} E(t)=\infty
$$

- Unter der zusätzlichen Voraussetzung (10) können wir nun dem hinreichenden Kriterium für die Existenz einer positiven Lösung von $(1 \Rightarrow)$ aus Satz 7 ein notwendiges an die Seite stellen. Zunächst beweisen wir zwei Hilfssätze.

Für jede final positive Lösung $g$ von $(1 \leqq)$ und jede final positive Lösung $f$.von ' $(1 \geqq)$ sei

$$
l(g)=\varliminf_{t \rightarrow \infty} \frac{g(t-\delta(t))}{g(t)} \text { und } L(f)=\varlimsup_{t \rightarrow \infty} \frac{f(t-\Delta(t))}{f(t)}
$$

Wir werden beim Beweis von Lemma 1 leicht sehen, daß eine final positive Lösung $g$ von $(1 \leqq)$ auch final monoton fallend ist. Daher ist $l(g) \geqq 1$.

Lemma 1: Sei $n(\infty)>0$ und sẹ geine final positive Lösung von $(1 \leqq)$. Dann gilt $l(g)<\infty$.

Beweis: Wegen $n(\infty)>0, g(t)>0$ für hinreichend große $t$ und wegen (11) können wir $T>A$ und $c>0$ so wählen, $\operatorname{da} \beta E(E(T)) \geqq A$ und .

$$
\begin{aligned}
& 0<c \leqq \int_{t-\delta(t)}^{t} M(s) d s \quad(E(T) \leqq t), \\
& 0<g(t) .(E(E(E(T))) \leqq t),
\end{aligned}
$$

ist. Wegen (13) erhalten wir für $t \geqq E(E(T))$

$$
g^{\prime}(t) \leqq-\int_{i=0}^{\infty} g(t-\tau) d r(t, \tau) \leqq 0 .
$$

Also ist $g$ für $t \geqq E(E(T))$ monoton fallend, und aus $(1 \leqq)$ folgt

$$
\dot{g}^{\prime}(t) \leqq-M(t) g(t-\delta(t))(E(T) \leqq t) .
$$


Wir wählen nun zu jedem $t \geqq T$ zwei Werte $t_{1}$, $t_{2}$, so daß $t-\delta(t)<t_{2}<t_{1}<t$ und

$$
\int_{t_{1}}^{t} M(s) d s=\int_{t_{1}}^{t_{1}} M(s) d s=\frac{c}{4}
$$

ist. Diese Wahl von $t_{1}, t_{2}$ ist wegen (12) möglich. Für alle $s \in\left[t_{1}, t\right]$ gilt dann

$$
s-\delta(s) \leqq t_{2}
$$

Würde nämlich für ein $s^{*} \in\left[t_{1}, t\right]$ gelten $s^{*}-\delta\left(s^{*}\right)>t_{2}$, so wäre

$$
\int_{s^{*}-\delta\left(s^{*}\right)}^{s^{*}} M(s) d s \leqq \int_{i,}^{t} M(s) d s=\frac{c}{2}
$$

im Widerspruch zu (12). Beachten wir (14), (16) und die Monotonie von $g$ auf $[E(E(T))$,$\infty)$, so erhalten wir $g^{\prime}(s) \leqq-M(s) g\left(t_{2}\right)\left(t_{1} \leqq s \leqq t\right)$ und damit

$$
g(t)-g\left(t_{1}\right) \leqq-g\left(t_{2}\right) \int_{t_{1}}^{t} M(s) d s=-g\left(t_{2}\right) \cdot \frac{c}{4}
$$

für $t \geqq T$. Hieraus folgt, da $g(t)$ positiv ist,

$$
\frac{g\left(t_{2}\right)}{g\left(t_{1}\right)}<\frac{4}{c} \quad\left(T^{\prime} \leqq t\right) .
$$

Wir nehmen nun an, die Behauptung sei nicht richtig. Dann ist $l(g)=\infty$, und da außerdem (11) gilt, können wir ein $T_{1}>T$ wählen, so da $B$

$$
\frac{g(t-\delta(t))}{g(t)} \geqq \frac{16}{c^{2}} \quad\left(E\left(T_{1}\right) \leqq t\right)
$$

ist. Dividieren wir (14) durch $g(t)$, beachten (18) und integrieren, so erhalten wir

$$
\ln \frac{g\left(t_{1}\right)}{g\left(t_{2}\right)} \leqq-\frac{16}{. c^{2}} \cdot \int_{t_{2}}^{t_{1}} M(s) d s \quad\left(T_{1} \leqq t\right) .
$$

Wegen (15) ist also

$$
\frac{g\left(t_{2}\right)}{g\left(t_{1}\right)} \geqq \ln \frac{g\left(t_{2}\right)}{g\left(t_{1}\right)} \geqq \frac{4}{c} \quad\left(T_{1} \leqq t\right)
$$

im Widerspruch .zu (17)

Lem ma 2: Für jede final positive Lösung g von $(1 \leqq)$ gilt

$$
\frac{\ln l(g)}{l(g)} \geqq n(\dot{\infty}) \text {. }
$$

Bewe is: Im Fall $l(g)=\infty$ muß nach Lemma 1 gelten $n(\infty)=0$. Also ist $(20)$ in diesem Fáll erfüllt. Sei nun $l(g)<\infty$. Zu beliebigem $\varepsilon>0$ wählen wir dann ein $T>A$, 
so $\operatorname{da} B \dot{E}(E(T)) \geqq A$ und

$$
\begin{aligned}
& l(g)-\varepsilon \leqq \frac{g(t-\delta(t))}{g(t)} \quad(E(T) \leqq t), \\
& 0<g(t) \quad(E(E(E(T))) \leqq t)
\end{aligned}
$$

ist. Wir haben dabei wieder (11) benutzt (im folgenden wird (11) noch oft benutzt, ohne daß wir darauf hinweisen). Wie beim Beweis von Lemma 1 erhalten wir aus (22)

$$
\bar{g}^{\prime}(t) \leqq-M(t) g(t-\delta(t)) \quad\left(E\left(T^{\prime}\right) \leqq t\right) .
$$

Dividieren wir (23) durch $g(t)$, beachten (21) und integrieren, so erhalten wir

$$
\ln \frac{g(t-\delta(t))}{g(t)} \geqq \int_{t \div \delta(t)}^{t} M(s) d s \cdot[l(g)-\varepsilon] .(T \leqq t) .
$$

Lassen wir hierin $t \rightarrow \infty$ gehen, so folgt $\ln l(g) \geqq n(\infty)(l(g)-\varepsilon)$. Da $\varepsilon>0$ beliebig war, ist (20) bewiesen

Wegen $\max _{0<\lambda \leq \infty} \frac{\ln \hat{\lambda}}{\lambda}=\frac{1}{\mathrm{e}}$ folgt aus Lemma 2 unmittelbar die folgende notwendige Bedingung für diè Existenz final pòsitiver Lösungen von $(1 \leqq)$.

Satz 8: Wenn $(1 \leqq)$ eine final positive Lösung besitzt, so, gilt $n(\infty) \leqq \frac{1}{\mathrm{e}}$.

Für die Gleichung $(\mathbf{1}=)$ kann man eine nichtoszillierende Lösung o. B. d. A. als final positiv annehmen. Daher erhält man aus Satz 8 das folgende Oszillationskriterium.

Satz $9: \operatorname{Bei} n(\infty)>\frac{1}{\mathrm{e}}$ besitzt $(1=)$ nur oszillierende Lösungen.

Anmerkungen: 1. Nach [13: Th. 49, Bem. 1] hat $(1=)$ bei $\lim _{t \rightarrow \infty}(t-\Delta(t))=\infty$ und $\frac{1}{\mathrm{e}}<m_{0} \delta_{0}$ nur oszillierende Lösungen. Wegen $n(\infty) \geqq m_{0} \delta_{0}$ ist dies in Satz 9 als Spezialfall enthalten. Vgl. auch Bemerkung 2 zu [13: Th. 49].

2. Es gibt eine Reihe von Oszillationskriterien für Spezialfälle von $(1=)$, s. z. B. $[9-11,14]$. Wir haben hier bei der Behandlung des allgemeinen Falles $(1 \doteq)$ in ähnlicher Weise geschlossen wie in $[10,11]$. Für den Spezialfall von $(1=)$ '

$$
\begin{aligned}
& y^{\prime}(t)=-\sum_{i=1}^{n} p_{i}(t) y\left(g_{i}(t)\right) \quad(A \leqq t), \\
& p_{i}(t), g_{i}(t) \quad \text { stetig auf }[A, \infty), \quad 0 \leqq p_{i}(t) \quad(A \leqq t), \\
& g_{i}(t) \leqq t \quad(A \leqq t), \quad \lim _{t \rightarrow \infty} g_{i}(t)=\infty \quad(i=1,2, \ldots, n)
\end{aligned}
$$

folgt auś Satz 9 sofort das Kriterium àus [11], da B (24) bei

$$
\varliminf_{t \rightarrow \infty}\left(\sum_{i=1}^{n} \int_{g^{*}(t)}^{t} p_{i}(s) d s\right)>\frac{1}{\mathrm{e}}, g^{*}(t)=\max _{1 \leqq i \leqq n} g_{i}(t)
$$

nur oszillierende Lösungen hat. Dabei wird hier die Monotonie der $g_{i}(t)$, die $e_{\triangleleft}$, [11] vorausgesetzt wurde, nicht benötigt. 
Wir führen ohne Beweis noch das folgende Analogon zu Lemma 2 an.

Lem ma 3: Für jede final positive und final monoton fallende Lösung f von (1 $\geqq$ gilt

$$
\therefore \frac{\ln L(f)}{L(f)} \leqq N(\infty) \text {. }
$$

Die Ungleichungen (20) und (25) liefern auf folgende Weise Abschätzungen für $l(g)$ und $\dot{L}(\dot{f})$. Für $0<p<\frac{1}{\mathrm{e}}$ hat die Gleichung $\frac{\ln \lambda}{\lambda}=p$ genau zwei positive Lösungen $\lambda_{1}(p)$ und $\lambda_{2}(p), 1<\lambda_{1}(p)<\mathrm{e}<\lambda_{2}(p)$. Im Fall $p=\frac{1}{\mathrm{e}}$ wird $\lambda_{1}(p)=\lambda_{2}(p)$ $=$ e eine Doppelwurzel. Besitzt nun $(1 \leqq)$ eine final positive Lösung $g$, so ist $n(\infty)$ $\leqq \frac{1}{\mathrm{e}}$. Ist außerdem $n(\infty)>0$, so sind also $\lambda_{1}(n(\infty))$ und $\lambda_{2}(n(\infty))$ definiert, und für $l(g)$ erhält man aus (20) die Abschätzung

$$
\lambda_{1}(n(\infty)) \leqq l(g) \leqq \lambda_{2}(n(\infty)) .
$$

Ist $f$ eine final positive und final monoton fallende Lösung von $(1 \geqq)$ und gilt $0<N(\infty) \leqq \frac{1}{\mathrm{e}}$, so folgt aus $(25)$ : Für $L(f)$ gilt

$$
L(f) \leqq \lambda_{1}(N(\infty)) \text { oder } \lambda_{2}(N(\infty)) \leqq L(f) .
$$

\section{Existenz positiver Lösungen von $(1=)$} mit unterschiedlichem asymptotischem Verhalten

Wir setzen voraus, da $\dot{B}(1=)$ eine positive Lösung besitzt, und betrachten die Menge $M$ aller Lösungen von $(1=)$. Die Aussage von Satz 1 gilt dann speziell für die Lösungen $/$ von $(1=): M$ ist dann also ein lineares Funktionensystem vom Durchdringungstyp (s. $[7,12])$. Erfüllt der Kern die zusätzliche Voraussetzung:

Für jedes $T \geqq A$ und jede auf $[E(T), T]$ stetige Funktion $\psi$ mit $\psi(t)<0(E(T) \leqq t<T)^{\prime}$ ist $\int_{r=0}^{\infty} \psi(T-\tau) d r(T, \tau)<0$,

so gilt für jede Lösung $f$ von $(1 \geqq)$ die folgende Aussage:

Aus $A \leqq T, f(t)<0(E(T) \leqq t<T)$ und $f(T)=0$ folgt $f(t)>0(T<t)$.

Wegen $(1 \geqq)$ und $(26)$ ist dann nämlich $f_{0}^{\prime}(T) \geqq-\int_{t=0}^{\infty} f(T-\tau) d r(T, \tau)>0$, so daß nach dem Zusātz zu Satz 1 tatsëchlich $f(t)>0\left(T^{\prime}<t\right)$ fölgt. Speziell gilt (27) für die Lösungen $f$ von $(1=), M$ ist also streng durchdringend (s. [7]). Hinreichend für (26) ist $0<M(t) \delta(t)(A \leqq t)$. Wir erhalten nun die folgendẹ Verschärfung der Sätze 4-7.

Satz 10: Ist die Voraussetzung eines der Sätze 4-7 erfüllt und gilt (26), so hat $(1=)$ zwei positive Lösungen $f$, und $g$ mit $g=o(f)$.

Beweis: (1 =) besitzt eine positive Lösung únd $M$ ist, da (26) gilt, streng durchdringend. Daher folgt die Behauptung unmittelbar aus [7: Satz 6] 
Anmerkungen: 1. Die Landauschen Symbole $O$ und $O$ verwenden wir hier und im folgenden stets für die Bewegung $t \rightarrow \infty$. Satz 10 ist eine Verallgemeinerung von [13: Th. 44].

2. Um [7: Satz 6] anwenden zu können, hätten wir eigentlich noch $\operatorname{dim} M^{\prime}>1$ nachprüfen müssen. Man sieht aber sofort, daß diese Voraussetzung dort nicht gebraucht wird, weil sie schon aus der Voraussetzung, da $B$ streng durchdringend ist, folgt.

\section{Klasseneinteilung}

Besitzt $(1 \doteq)$ final positive Lösungen, so nennen wir, wie üblich, zwei final positive Lösungen $f$ und $g \ddot{a} q u \dot{z} v a l e n t$, wenn $f=O(g)$ und $g=O(f)$ gilt. In der Menge der final positiven Lösungen von $(1=)$ ist damit eine Äquivalenzrelation definiert, sie zerfällt also in Klassen äquivalenter Elemente. Nach [7: Satz 3] existieren höchstens zwei Āquivalenzklassen. Sind zwei Klassen vorhanden, so ist nach [7: Satz 2] eine der Klassen, wir bezeichnen sie mit $X_{1}$, größer als andere, die wir mit $X_{2}$ bezeichnen. Dies bedeutet, daß für jede Lösung $x_{1} \in X_{1}$ und jede Lösung $x_{2} \in X_{2}$ gilt $x_{2}=o\left(x_{1}\right)$. Wir schreiben dafür $X_{2}<\dot{X}_{1}$.

Unter den Voraussetzungen von Satz 10 besitzt $(1=)$ positive Lösungen, die nicht äquivalent sind. $(1=)$ hat bei diesen Voraussetzungen also zwei Klassen. Im folgenden setzen wir voraus, daß $(1=)$ zwei Klassen besitzt und schätzen die final positiven Lösungen von $Y(1=)$ mit Hilfe der Funktionen $\varphi_{c}(t)=\exp \left[-c \cdot \int_{i}^{t} M(s) d s\right]$ ab.

\section{Abschätzung der final positiven Lösung von $(1=)$}

Nach $[8:$ Th. 4, 5] gilt der folgende Satz.

Satz 11 : $(1=)$ möge zwei Klassen final positiver Lösungen $X_{2}<X_{1}$ besitzen. Sei $x_{1} \in X_{1}, x_{2} \in X_{2}$. Weiter sei $f$ eine final positive Lösung von $(1 \geqq)$ und geine final positive Lösung von ( $1 \leqq)$. Dann gill:

1. Entweder ist $x_{1}=O(f)$ oder $f=O\left(x_{2}\right)$.

2. Es ist $\quad x_{2}=O(g)$ und $g=O\left(x_{1}\right)$.

Der folgende Satz ist eine direkte Anwéndung von Satz 11.

Satz $12:\left(1^{-}=\right)$möge zwei Klassen final positiver Lösungen $\dot{X}_{2}<X_{1}$ besitzen. Sei $x_{1} \in X_{1}, x_{2} \in X_{2}$. Weiter möge ein $T \geqq A$ existieren, so da $\beta$

$$
0<n(T) \leqq N(T)<\frac{1}{\mathrm{e}}
$$

ist. Die Zahlen $c_{1}, c_{2}, \tilde{c}_{1}, \tilde{c}_{2}$ seien so gewiihlt, daß

$$
1 \leqslant \tilde{c}_{1}^{\prime} \leqq c_{1}<c_{2} \leqq \tilde{c}_{2}
$$

und

$$
\frac{\ln \tilde{c}_{i}}{\tilde{c}_{i}} \leqq n(T) \leqq N(T) \leqq \frac{\ln c_{i}}{c_{i}} \quad(i=1,2)
$$


gilt. Dann folgt

sowie

$$
\varphi_{c_{1}}=O\left(x_{1}\right) . \text { und } x_{1}=O\left(\varphi_{\tilde{c}_{1}}\right)
$$

$$
\text { - } \varphi_{\tilde{c}_{2}}=O\left(x_{2}\right) \text { und } x_{2}=O\left(\varphi_{c_{3}}\right) \text {. }
$$

Anmerkungen: 1. Die Existenz von Zahlen $c_{1}, c_{2}, \tilde{c}_{1}$ und $\tilde{c}_{2}$, die den Bedingungen (29) und (30) genügen, folgt aus der Voraussetzung (28).

2. Aus (29)-erhalten wir die Beziehungen

$$
\varphi_{\tilde{c}_{2}}=O\left(\varphi_{c_{2}}\right)^{;}, \quad \varphi_{c_{2}}=o\left(\varphi_{c_{1}}\right), \quad \varphi_{c_{2}}=O\left(\varphi_{\tilde{c}_{1}}\right) .
$$

Um die $o$-Beziehung zu sehen, müssen wir beachten, da $\beta$ wegen $0<n(T)$ offenbar $\infty$

$\int M(s) d s=\infty$ gilt. Die Beziehung $\varphi_{c_{2}}=o\left(\varphi_{c_{1}}\right)$ zeigt, da $\beta$ die Lösungen $x_{1} \in X_{1}$ und $x_{2} \in X_{2}$ durch die Beziehungen $\varphi_{c_{1}}=O\left(x_{1}\right)$ und $x_{2}=O\left(\varphi_{c_{2}}\right)$ asymptotisch echt voneinander getrennt werden.

Beweis von Satz 12: Nach Anmerkung $1 \mathrm{zu}$ Satz 7 erfüllén für $t \geqq I^{\prime}$ die $\varphi_{c_{\mathrm{r}}}$ die die Ungleichung, $(1 \leqq)$ und die $\varphi_{\tilde{c}_{\mathfrak{r}}}$ die Ungleichung $(1 \geqq)$. Satz $11 / 2$ liefert also sofort

und

$$
\text { ; } \varphi_{c_{1}}=O\left(x_{1}\right)
$$

$$
x_{2}=O\left(\varphi_{c_{2}}\right) \text {. }
$$

Nach Satz 11/1 gilt entweder $x_{1}=O\left(\varphi_{\tilde{c}_{1}}\right)$ oder $\varphi_{\tilde{c}_{1}}=O\left(x_{2}\right)$. Wäre $\varphi_{\tilde{c}_{1}}=O\left(x_{2}\right)$, so wäre wegen $\varphi_{c_{3}}=o\left(\varphi_{c_{1}}\right)$ auch $\varphi_{c_{2}}=o\left(x_{2}\right)$, was im Widerspruch zu (34) steht.. Also gilt $x_{1}=O\left(\varphi_{\tilde{c}_{1}}\right)$. Für $\varphi_{\tilde{c}_{1}}$ liefert Satz 11/1 wieder: Entweder ist $x_{1}=O\left(\varphi_{\tilde{c}_{2}}\right)$ oder $\dot{\varphi}_{\bar{c}_{2}}$ $=O\left(x_{2}\right)$. Wäre $x_{1}=O\left(\varphi_{\tilde{c}_{2}}\right)$, so wäre wegen $\varphi_{\tilde{c}_{2}}=o\left(\varphi_{c_{1}}\right)$ auch $x_{1}=o\left(\varphi_{c_{1}}\right)$ im Widerspruch zu (33): Also gilt $\varphi_{\bar{c}_{2}}=O\left(x_{2}\right)$

Satz 11 ist für Lösungen von (1 ) formuliert, nach unserer Festlegung des Lösungsbegriffes also für Funktionen, die (15) von $A$ an erfüllen. Wir können ihn aber natür lich auf die Funktionen $\varphi_{c_{t}}$ bzw. $\varphi_{\tilde{c}_{i}}$, die $(1 \leqq)$ bzw. $(1 \geqq)$ erst von $T$ an erfüllen; anwenden. Dazu brauchen wir nur den Anfangspunkt nach $T$ zu verlegen. Entsprechendes gilt auch im folgenden.

Die folgenden beiden Sätze verschärfen Satz 12. Zunächst befássen wir uns mit der Verschärfung der Relationen (31).

Satz 13: Unter den Voraussetzungen des Satzes 12 existieren endliche Grenzwerte

$$
\lim _{t \rightarrow \infty} \frac{x_{1}(t)}{\varphi_{\tilde{c}_{1}}(t)} \quad \text { und } \quad \lim _{t \rightarrow \infty} \frac{\varphi_{c_{1}}(t)}{x_{1}(t)}
$$

Wir beweisen zunächst ein Lemma.

Lemma $4:$ f sei eine final positive Lösung von (1ฏ), zu der eine final positive Lösung $\tilde{g}$ von $(1 \leqq)$ existieren möge mit $\tilde{g}=o(f)$. Ist dann $g$ eine final positive Lösung von $(1 \leqq)$, so konvergiert $\frac{g(t)}{f(t)}$ für $t \rightarrow \infty$ gegen einen endlichen Grenzwert.

Beweis: Wir wählen ein $T_{1} \geqq A$, so daß $f(t), g(t)$ und $\tilde{g}(t)$ für $t \geqq E\left(T_{1}\right)$ positiv sind. Weiter wählèn wir ein $\alpha>0$, so daß

$$
f(t)<\alpha \tilde{g}(t) \quad\left(E\left(T_{1}\right) \leqq t \leqq T_{1}\right)
$$


ist. Es existiert ein $T_{2}>T_{1}$ mit $f\left(T_{2}\right)>\alpha \tilde{g}\left(T_{2}\right)$, da andernfalls $f=O(\tilde{g})$ wäre im Widerspruch zur Voraussetzung. Wir wählen ein $\beta>0$, so daß auch noch gilt $f\left(T_{2}\right)$ $>\alpha \tilde{g}\left(T_{2}\right)+\beta g\left(T_{2}\right)$. Wegen (36) ist außerdem $f(t)<\alpha \tilde{g}(t)+\beta g(t)\left(E\left(T_{1}\right) \leqq t \leqq T_{1}\right)$. Ist daher $T_{3}=\inf \left\{t: t>T_{1}\right.$ und $\left.f(t)>\alpha \tilde{g}(t)+\beta g(t)\right\}$, so gilt $T_{1}<T_{3}$ und

$$
\left.\begin{array}{l}
f(t) \leqq \alpha \tilde{g}(t)+\beta g(t)\left(E\left(T_{3}\right) \leqq t \leqq T_{3}\right) \\
f\left(T_{3}\right)=\alpha \tilde{g}\left(T_{3}\right)+\beta g\left(T_{3}\right)
\end{array}\right\}
$$

Nach Satz 2 ist also der Quotient $\frac{f(t)}{\alpha \tilde{g}(t)+\beta g(t)}$ auf $\left[T_{3}, \infty\right)$. monoton wachsend.
Daher konvergiert

$$
\frac{\alpha \tilde{g}(t)+\beta g(t)}{f(t)}
$$

für $t \rightarrow \infty$ gegen einen endlichen Grenzwert. Wegen $\tilde{g}=o(f)$ folgt hieraus die Behaup-, tung'

Beweis von Satz 13: Wenn wir Lemma 4 anwenden mit $f=\varphi_{\tilde{c}_{1}}, g=x_{1}$ und $\dot{\bar{g}}=x_{2}$, so erhalten wir die Existenz des ersten der Girenzwerte (35). $\bar{g}=o(f)$ ist erfüllt, da $x_{2}=o\left(x_{1}\right)$ und nach Satz $12 x_{1}=O\left(\varphi_{\bar{c}_{1}}\right)$ gilt. Wenden wir Lemma 4 mit $f=x_{1}, g=\varphi_{c_{1}}$ und $\tilde{g}=x_{2}$ an, erhalten wir die Existenz des zweiten Grenzwertes

Nun verschärfen wir die Relationen (32).

Satz 14: Unter den Voraussetzungen des Satzes 12 existieren endliche Grenzwerte

$$
-\lim _{t \rightarrow \infty} \frac{x_{2}(t)}{\varphi_{c_{2}}(t)} \text { und } \lim _{t \rightarrow \infty} \frac{\varphi_{\tilde{t}_{z}}(t)}{x_{2}(t)}
$$

Bevor.wir Satz 14 beweisen, stellen wir drei Hilfssätze bereit. Dabei sei $h^{+}$für eine Funktion $h$ die durch $h^{+}(t)=\max (h(t), 0)$ definierte Funktion.

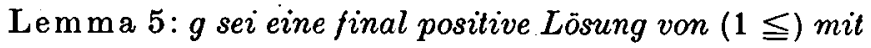

$$
\lim _{t \rightarrow \infty} \frac{g(t)}{g(E(t))}>0
$$

Dann gilt:

1. Ist $h$ eine oszillierende Lösung von $(1 \geqq)$, so ist $h^{+}=o(g)$. 2. Ist f eine final positive Lösung von $(1 \geqq)$ mit $f=O(g)$, so konvergiert $\frac{f(t)}{g(t)}$. für
$t \rightarrow \infty$ gegen einen endlichen Grenzwert.

Beweis: 1. Wegen (40) können wir eine Stelle $S>A$ und eine Zahl $k>0$ wählen, so $\operatorname{da} B 0<g(t)(E(S) \leqq t)$ und

$$
\frac{g(t)}{g(E(t))}>k>0 \quad(S \leqq t)
$$

gilt. Wegen $0<g(t)(E(S) \leqq t)$ ist $g$ auf $[S, \infty)$ monoton fallend. Sei nun $\alpha>0$ so gewählt, daß $h(t)<\alpha g(t)(E(S) \leqq t \leqq S)$ ist. Diese Ungleichung bleibt für alle $t \geqq S$ bestehen. Wäre das nämlich nicht der Fall, so gäbe es eine kleinste Stelle $T_{1}>S$ mit $h\left(T_{1}\right)=\alpha g\left(T_{1}\right)$. Für diese Stelle wäre dann auBerdem $h(t)<\alpha g(t)\left(E\left(T_{1}\right) \leqq t<T_{1}\right)$. Nach Satz 2 würde also $h(t) \geqq \alpha g(t)>0\left(T_{1} \leqq t\right)$ folgen - im Widerspruch dazu, daß 
$h$ oszilliert. Also gilt tatsächlich

$$
h(t) \dot{<} \alpha g(t)(E(S) \leqq t)
$$

Da $h$ oszilliert, können wir ein $S_{1}$ wählen, so daß $E\left(S_{1}\right) \geqq S$ und $h\left(S_{1}\right)=0$ ist; Wir zeigen, daß dann sogar

$$
h(t)<\alpha(1-k) \dot{g}(t)\left(E\left(S_{1}\right) \leqq t\right)
$$

gilt. Dazu betrachten wir die Lösung $g_{1}=\alpha g-h$. von $(1 \leqq)$. Da sie auf $[E(S), \infty)$ positiv ist, ist sie auf $[S, \infty)$ monoton fallend. Wegen $E\left(S_{1}\right) \geqq S$ ist $g_{1}$ speziell auf [ $\left.E\left(S_{1}\right), S_{1}\right]$ fallend. Also gilt für $t \in\left[E\left(S_{1}\right), S_{1}\right]$ die Ungleichung $g_{1}(t) \geqq g_{1}\left(S_{1}\right)$, wegen $h\left(S_{1}\right)=0$ also $\alpha g(t)-h(t) \geqq \alpha g\left(S_{1}\right)$. Also wird für $t \in\left[E\left(S_{1}\right), S_{1}\right]$

$$
h(t) \leqq \alpha\left(g(t)-g\left(S_{1}\right)\right)=\alpha\left(1-\frac{g\left(S_{1}\right)}{g(t)}\right) g(t)
$$

woraus wegen der Monotonie von $g$ und (41) folgt

$$
h(t) \leqq \alpha\left(1-\frac{g\left(S_{1}\right)}{g\left(E\left(S_{1}\right)\right)}\right) g(t)<\alpha(1-k) g(t) .
$$

Damit ist (43) für alle $t \in\left[E\left(S_{1}\right), S_{1}\right]$ als richtig erkannt. Wie oben sieht man, daß (43) für $t \geqq S_{1}$ bestehen bleibt. Durch wiederholte Anwendung der eben benutzten SchluBweise erhalten wir: Zu jeder natürlichen Zahl $i$ existiert ein $S_{i}$, so daß gilt

$$
h(t)<\alpha(1-k)^{i} g(t) \dot{(}\left(E\left(S_{i}\right) \leqq t\right) .
$$

Damit ist die erste Behauptung bewiesen.

2. Wegen $f=O(g)$ ist $0 \leqq c=\varliminf_{t \rightarrow \infty} \frac{f(t)}{g(t)} \leqq \varlimsup_{t \rightarrow \infty} \frac{f(t)}{g(t)}=C<\infty$. Wäre $c<C$, so wäre $h=f-\frac{c+C}{2} g$ eine oszillierende $i_{t \rightarrow \infty} g(t)$ ' $=\varlimsup_{t \rightarrow \infty} \frac{f(t)}{g(t)}-\frac{c+C}{2}=\frac{C-c}{2}>0$ gilt, was im Widerspruch zu Behauptung 1 steht. Also muß $c=\dot{C}$ sein. Damit ist auch Behauptung 2 bewiesen

Anmerkung: Mit $h$ ist auch $-h$ eine oszillierende Lösung von $(\mathbf{1}=)$. Für die oszillierenden Lösungen $h$ von $(1=)$ folgt daher aus Behauptung 1 sofort

$$
\therefore \quad h=o(g) \text {. }
$$

Wollen wir Iemma 5 anwenden, müssen wir wissen, daß $g$ die Voraussetzung (40) erfüllt. Dazu beweisen wir die fọlgenden zẃei Lemmata.

Le m ma-6: Es mögen eine final positive und final monoton fallende Lösung f von (1 $\geqq)$ und eine final positive Lösung $\tilde{g}$ von $(1 \leqq)$ existieren, so daß $f=o(\tilde{g})$ ind

$$
\varliminf_{t \rightarrow \infty} \frac{f(t)}{f(E(T))}>0
$$

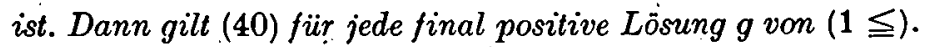

Beweis: Wir wählen ein $T_{1}$ mit $E\left(E\left(T_{1}\right)\right) \geqq A$, so dàß $f, g$ und $\tilde{g}$ auf $\left[E\left(E\left(E\left(T_{1}\right)\right)\right)\right.$, $\infty)$ positiv sind und $f$ auf diesem Intervall monoton fällt. $g$ und $\tilde{g}$ sind dann auf 
$\left[E\left(E\left(T_{1}\right)\right), \infty\right)$ auch monoton fallend. Sei nun eine' beliebigé Stelle $T_{2} \geqq T_{1}$ gewählt und

Dann ist

$$
\gamma=\max \left\{\frac{f(t)}{g(t)}: \dot{E}\left(E\left(T_{2}\right)\right) \leqq t \leqq E\left(T_{2}\right)\right\} .
$$

$$
f(t) \leqq \gamma g(t)\left(E\left(E\left(T_{2}\right)\right) \leqq t \leqq \dot{E(}\left(T_{2}\right)\right),
$$

und es existiert ein $P \in\left[E\left(E\left(T_{2}\right)\right), E\left(T_{2}\right)\right]$ mit

$$
f(P)=\gamma g(P) \text {. }
$$

Für alle $t \geqq E\left(T_{2}\right) \mathrm{mu} B$ gelten

$$
f(t) \leqq \gamma g(t) \text {. }
$$

Nehmen wir nämlich an, daß für ein $T_{3} \geqq E\left(T_{2}\right)$ gilt $f\left(T_{3}\right)>\gamma g\left(T_{3}\right)$, so können wir ein $\beta>0$ wählen, so 'da $\beta$ auch noch gilt $f\left(T_{3}\right)>\gamma g\left(T_{3}\right)+\beta \bar{g}\left(T_{3}\right)$. Außerdem ist wegen (48) $f(t)<\gamma g(t)+\beta \tilde{g}(t) \quad\left(E\left(E\left(T_{2}\right)\right) \leqq t \leqq E\left(T_{2}\right)\right)$. Ist daher $T_{4}=\inf \{t: t$ $>E\left(T_{2}\right)$ und $\left.f(t)>\gamma g(t)+\beta \tilde{g}(t)\right\} ;$ so ist $E\left(T_{2}\right)<T_{4}$ und

$$
\left.\begin{array}{l}
f(t) \leqq \gamma g(t)+\beta \tilde{g}(t)\left(E\left(T_{4}\right) \leqq t \leqq T_{4}\right) \\
f\left(T_{4}\right)=\gamma g\left(T_{4}\right)+\beta \bar{g}\left(T_{4}\right) .
\end{array}\right\}
$$

Nach Satz 2 folgt also $f(t) \geqq \gamma g(t)+\beta \tilde{g}(t)>\beta \tilde{g}(t)\left(T_{4} \leqq t\right)$ im Widerspruch zur Vor: aussetzung $f=o(\tilde{g})$. Also gilt für alle $t \geqq E\left(T_{2}\right)$ tatsächlich (5̦0). Aus (49) und (50) folgt nun $\frac{\gamma g(t)}{f(t)} \geqq \frac{\gamma g(P)}{\prime f(P)}\left(E\left(T_{2}\right) \leqq t\right)$. Bei spezieller Wahl $t=T_{2}$
erhalten wir also

$$
\frac{g\left(T_{2}\right)}{g(P)} \geqq \frac{f\left(T_{2}\right)}{f(P)}
$$

Wegen $E\left(E\left(T_{2}\right)\right) \leqq P \leqq E\left(T_{2}^{\prime}\right)$ und der Monotonie von $g$ und $f$ ist $g(P) \geqq g\left(E\left(T_{2}\right)\right)$ und $f(P) \leqq f\left(E\left(E\left(T_{2}\right)\right)\right)$. Daher folgt aus (52) .

$$
\frac{g\left(T_{2}\right)}{g\left(E\left(T_{2}\right)\right)} \geqq \frac{f\left(T_{2}\right)}{f\left(E\left(E\left(T_{2}\right)\right)\right)}=\frac{f\left(T_{2}\right)}{f\left(E\left(T_{2}\right)\right)} \cdot \frac{f\left(E\left(T_{2}\right)\right)}{f\left(E\left(E\left(T_{2}\right)\right)\right)}
$$

Da (53) für jede Stelle $T_{2} \geqq T_{1}$ gilt, folgt aus.(53) und (46) die Behauptung

Lemma 7: Gilt

$$
0<n(\infty) \leqq N(\infty)<\infty,
$$

so ist (40) für jede final positive Lösung g von $(1 \leqq)$ erfüllt.

Beweis: Wegen (54) können wir ein $T_{1} \geqq A$ so wählen, daß $0<n\left(T_{1}\right) \leqq N\left(T_{1}\right)$ $<\infty$ ist. Weiter können wir wegen $N\left(T_{1}\right)<\infty$ ein $K>0$ wählen mit

$$
\int_{E(t)}^{t} M(s) d s \leqq K \quad\left(T_{1} \leqq t\right) .
$$

Nun wählen wir eine Zahl $0<k<1$, so da $B$

$$
0<k \cdot n\left(T_{1}\right) \leqq \dot{k} \cdot N\left(T_{1}\right)<\frac{1}{\mathrm{e}},
$$


ist, und betrachten auf $\left[T_{1}, \infty\right)$ die Ungleichung

$$
x^{\prime}(t) \leqq-\int_{\tau=0}^{\infty} x(t-\tau) d(k r(t, \tau)) \quad\left(T_{1} \leqq t\right)
$$

mit dem Kern $k \cdot r(t, \tau)$. Wegen (56) können wir Zahlen $d_{1}, d_{2}$ so wählen, da $\beta 1<d_{1}$ $<d_{2}$ und

$$
\frac{\ln d_{2}}{d_{2}} \leqq k n\left(T_{1}\right) \leqq k N\left(T_{1}\right) \leqq \frac{\ln d_{1}}{d_{1}}
$$

gilt. Dann ist $f=\varphi_{d_{3}}$ Lösung von $\left(1_{k} \geqq\right)$ und $\tilde{g}=\varphi_{d_{1}}$ Lösung von $\left(1_{k} \leqq\right)$. Wegen $0<k n\left(T_{1}\right)$ und $d_{1}<d_{2}$ gilt $\varphi_{d_{1}}=o\left(\varphi_{d_{1}}\right)$, d. h. $f=o(\tilde{g})$. Außerdem ist wegen (55)

$$
\varliminf_{t \rightarrow \infty} \frac{f(t)}{f(E(t))}=\varliminf_{t \rightarrow \infty} \exp \left(-d_{2} \int_{E(t)}^{t} M(s) d s\right)>0 \text {. }
$$

Also ist Lemma 6 anwendbar und wir erhalten, dá $B$ für jede final positive Lösung $g$ von $\left(1_{k} \leqq\right)$ die Beziehung (40) gilt. Ist nun $g_{0}$ eine final positive Lösung von $(1 \leqq)$, so existiert ein $T_{2}>T_{1}$ mit $0<g_{0}(t)\left(E\left(T_{2}\right) \leqq t\right)$, und wegen $0<k<1$ folgt aus ( $1 \leqq), \operatorname{da} B g_{0}$ für $t \geqq T_{2}$ die Ungleichung $\left(1_{k} \leqq\right)$ erfüllt. Also gilt (40) für $g_{0}$

Anmerkung: Besitzt ( $1 \leqq$ ) eine final positive Lösung $g$ und gilt $0<m_{0} \delta_{0}, \Delta_{0}<\infty$, so ist (54) erfüllt. Denn wegen $m_{0} \delta_{0} \leqq n(\infty)$ ist dann natürlich $n(\infty)>0$. Zu zeigen ist also'nur $N(\infty)<\infty$. Dazu wählen wir ein $T_{1}$, so daß $0<g(t)\left(E\left(E\left(E\left(T_{1}\right)\right)\right) \leqq t\right)$ gilt. Dann ist $g$ auf $\left[E\left(E\left(T_{1}\right)\right), \infty\right)$ monoton fallend. Ist $E\left(T_{1}\right) \leqq \cdot s \leqq t$, so gilt also

$$
g^{\prime}(s) \leqq-\int_{\tau=0}^{\infty} g(s-\tau) d r(s, \tau) \leqq-M(s) g\left(s-\delta_{0}\right) \leqq-M(s) g\left(t-\delta_{0}\right) .
$$

Nach Integration erhält man hieraus leicht

Also ist

$$
0<g(t) \leqq g\left(t-\delta_{0}\right)\left[1-\int_{t-\delta_{0}}^{t} \boldsymbol{M}(s) d s\right] \quad\left(T_{1} \leqq t\right)
$$

$$
\int_{t-\delta_{0}}^{t} M(s) d s<1 \quad\left(T_{1} \leqq t\right)
$$

Sei $n$ eine natürliche Zahl mit $n \delta_{0}>\Delta_{0}$. Weiter sei $T_{2}=T_{1}+n \delta_{0}$. Dann gilt wegen (59) für $t \geqq T_{2}$

$$
\int_{t-\Delta(t)}^{t} M(s) d s \leqq \int_{t-\Delta_{0}}^{t} M(s) d s \leqq \int_{t-n \delta_{0}}^{t} M(s) d s=\int_{t-n \delta_{0}}^{t-(\bar{n}-1) \delta_{0}} M(s) d s+\cdots+\int_{t-\delta_{0}}^{t} \dot{M}(s) d s \leqq n .
$$

Wir erhalten also tatsächlich $N(\infty) \leqq N\left(T_{2}\right) \leqq n<\infty$.

Beweis von'Satz 14: Wegen (28) gilt nach Lemma 7 für jede final positive Lösung $g$ von $(1 \leqq)$ die Beziehung (40). Speziell gilt (40) also für $x_{2}$ und $\varphi_{c_{2}}$ (letzteres kann man auch, ohne Lemma 7 heranzuziehen, direkt sehen; vgl. (58)). Wenn wir Lemma 5/2 anwenden mit $f=x_{2}$ und $g=\varphi_{c_{2}}$, erhalten wir die Existenz des ersten der Grenzwerte (39). $f=O(g)$, d. h. $x_{2}=O\left(\varphi_{c_{2}}\right)$, gilt nach Satz 12. Wenden wir Lemma 5/2 an mit $f=\varphi_{\tilde{c}_{1}}$, und $g=x_{2}$, erhalten wir die Existenz des zweiten der Grenzwerte (39). $f=O(g)$, d. h. $\varphi_{\tilde{c}_{2}}=O\left(x_{2}\right)$, gilt wieder nach Saitz 12 
Die Sätze 12-14 sind Folgerungen aus Satz 11 und den Lemmata 4-6. Man kann aus Satz 11 und diesen Lemmata leicht weitere Ergebnisse, Analoga zu den Sätzen 12-14, ableiten, wenn man statt der $\varphi_{c}$ andere Lösungen von $(1 \leqq)$ bzw. (1 ) als Vergleichsfunktionen heranzieht. Insbesondere erhält man aus Satz 11 sofort [13: Th. 45]. UUberdies kann man mit den Lemmata 4-6 die Verschärfungen von [13: Th. 45] beweisen, die den Sätzen 13 und 14 entsprechen.

\section{Entwicklungssatz}

Wir beweisen zum Schluß den folgenden Entwicklungssatz.

Sàtz 15: $(1 \Rightarrow)$ möge zwei Klassen final positiver Lösungen $X_{2}<X_{1}$ besitzen. Weiter sei

$$
0<n(\infty) \leqq N(\infty)<\infty
$$

und $x_{1} \in X_{1}, x_{2} \in X_{2}$. Dann läßt sich jede Lösung $x$ von $(1=)$ in eindeutiger Weise darstellen als

$$
x=\alpha_{1} x_{1}+\alpha_{2} x_{2}+r_{2},
$$

wobei $\alpha_{1}, \alpha_{2}$ reelle Konstanten sind und $r_{2}=o\left(x_{2}\right)$ gill:

Beweis: Die Einzzigkeit der Darstellung ist offensichtlich. Wir zeigen die Existenz. Zunächst bemerken wir, da $B x_{1}$ und $x_{2}$ nach Lemma 7 der Beziehung (40) genügen.

1. Ist $x$ eine oszillierende Lösung von $(1=)$, so gilt nach der Anmerkung zu Lemma $5 x=o\left(x_{2}\right)$. Also können wir in (61) $\alpha_{1}=\alpha_{2}=0$ und $r_{2}=x_{2}$ setzen.

2. Sei nun $x$ nicht oszillierend. Wir nehmen o. B. d. A. an, daß $x$ final positiv ist.

Dann gilt $x \in X_{2}$ oder $x \in X_{1}$.
a) Ist $x \in X_{2}$, so gilt $x=O\left(x_{2}\right)$. Nach Lemma 5 konvérgiert also $\frac{x(t)}{x_{2}(t)}$ für $i \rightarrow \infty$ gegen einen endlichen Grenzwert. Setzen wir $\alpha_{1}=0, \alpha_{2}=\lim _{t \rightarrow \infty} \frac{x(t)}{x_{2}(t)}, r_{2}=x-\alpha_{2} x_{2}$
so erhalten wir die gesuchte Darstellung.

b) Ist $x \in X_{1}$, so gilt $x=O\left(x_{1}\right)$. Nach Lemma 5 konvergiert also $\frac{x(t)}{x_{1}(t)}$ für $t \rightarrow \infty$ gegen einen endlichen Grenzwert. Setzen wir $\alpha_{1}=\lim _{t \rightarrow \infty} \frac{x(t)}{x_{1}(t)}$ und $\tilde{x}=x-\alpha_{1} x_{1}$, so
wird also

$$
\dot{x}=\alpha_{1} x_{1}+\bar{x}, \quad \tilde{x}=o\left(x_{1}\right) \text {. }
$$

$\tilde{x}$ ist ebenfalls Lösung von ${ }^{-}(1=)$. Ist $\dot{x}$ oszillierend, so gilt $\tilde{x}=o\left(x_{2}\right)$.Also setzen wir' $\alpha_{2}=0, r_{2}=\tilde{x}$ und erhalten aus (62) die gesuchte Darstellung. Ist $\tilde{x}$ nicht oszillierend, so können wir o. B. d. A. annehmen, da $\beta \bar{x}$ final positiv ist. Wegen $\bar{x}=o\left(x_{1}\right)$ ist $\tilde{x} \in X_{2}$. Also können wir nach a) schreiben $\tilde{x}=\dot{\alpha}_{2} x_{2}+r_{2}, r_{2}=o\left(x_{2}\right)$. Setzen wir dies in (62) ein, so erhalten wir (61)

Anmerkung: In [3] wurde bewiesen, daß der Entwicklungssatz gilt, wobei an Sțelle von $(60)$ vorausgesetzt wurde: $0<m_{0} \delta_{0} \leqq \frac{1}{\mathrm{e}}, \Delta_{0}<\infty$ (vgl. auch [13: S. 203/204]). Dies ist in Satz 15 als Spezialfall enthalten, wie unmittelbar aus der Anmerkung, zu Lemma 7 folgt.. 


\section{LITERATUR}

[1] Kozakiewicz, E.: Uber das asymptotische Verhalten der nichtschwingenden Lösungen einer linearen Differentialgleichung mit nacheilendem Argument. Wiss. Z. Humb.-Univ. Berlin/Mat.-Nat. R. 13 (1964), 577-589.

[2] Kozakiewicz, E.: Zur Abschätzung des Abklingens der nichtschwingenden Lösurigen einer linearen Differentialgleichung mit nacheilendem Argument. Wiss. Z. Humb.-Univ. Berlin/Math.-Nat. R. 15 (1966), 675 -676.

[3] KozAkIEwicz, E.: Uber die nichtschwingenden Lösungen einer linearen Differentialgleichung mit nacheilendem Argument. Math. Nachr. 32 (1966), 107-113.

[4] Kozariewicz, E.: Uber einen Satz von Myschkis. Math. Nachr. 35 (1967), 235-240.

[5] Kozakiewicz, E.: Uber die Lage der Nullstellen gewisser Lösungen einer linearen Differentialgleichung mit nacheilendem Argument. Math. Nachr. 35 (1967), 149-152.

[6] Kozakiewicz, E.: Uber die Länge der Halbzyklen der Lösungen einer linearen Differentialgleichung mit nacheilendem Argument vom stabilen Typus. Wiss. Z. Humb.-Univ. -Berlin/Math.-Nat. R. 17 (1968), 703-704.

[7] Kozakiewicz, E.: Uber lineare Funktionensysteme vom Durchdringungstyp. Periodica Math. Hungar. 7 (1976), 37-48.

[8] Kozakiewicz, E.: On the asymptotic behaviour of positive solutions of two differential inequalities with retarded argument. In: Coll. Math. Soc. Jan. Bolyai 15: Differential Equations. Proc. Conf., Keszthely (Hurigary) 1975 (Ed. M. Farkas). Budapest: Jan. Bol. Math. Soc. 1977, 309-319.

[9] Ladas, G., Lakshoinantham, V., and J. S. Pिapadakis: Oscillations of higher - order retarded differential equations generated by the retarded argument. In: Delay and functional differential equations and their applications. Proc. Conf. Park City/Utah (USA), March 6-11, 1972 (Ed. Klaus Schmitt). New York and London: Academic Press 1972, $219-231$.

[10] Ladas, G.: Sharp conditions for oscillations caused by delays. Appl. Analysis 9 (1979), $93-98$.

[11] LADDE, G. S.: Oscillations caused by retarded perturbations of first order linear ordinary differential equations. Atti Accad naz. Lincei, VIII Ser. Rend., Cl. sci. fis. mat. nat. 63 (1977), 351-359.

[12] Morgenthal, K.: Asymptotische Untersuchungen bei Funktionensystemen mit modifizierter Durchdringungseigenschaft. Beiträge z. Analysis 13 (1979), 179-189.

[13] Мышкис, А. Д..: Линейные дифференциальные уравнения с запаздывающим аргументом, изд. 2. Москва: Изд-во Наука 1972.

[14] Трлмов, М. И.: Условия колеблемости решений дифферепциальных уравнений первого порядка с запаздывающим аргументом. Изв. вузов/Сер. матем. 3 (1975), $92-96$.

[15] Щев Ело, В. Н., и Н. В. ВАРех: Об исследовании осцилляторныХ свойств решений дифференциальных уравнений первого порядка с отклоняющимся аргументом. В сб.: Асимптотические методы в теории нелинейны колебаний. Труды всесоюзной копф., Кацивели (СССР) 1977 (ред.: Ю. А. Митропольскин). Киев: Изд-во Наукова думка 1979, 247-262.

Manuskripteingang: 07. 10.1983

\section{VERFASSER:}

\section{Dr. Klaus Moroenthat}

Sektion Mathematik der Humboldt-Universität

DDR - 1086 Berlin, Unter den Linden 6 